Geometry $8 \mathcal{F}$ Topology

Volume 3 (1999) 155-165

Published: 2 July 1999

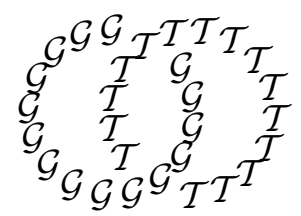

\title{
Vanishing lines in generalized Adams spectral sequences are generic
}

\author{
M J HOPKINS \\ J H PALMIERI \\ J H SMITH \\ Department of Mathematics, Massachusetts Institute of Technology \\ Cambridge, MA 02139, USA \\ Department of Mathematics, University of Notre Dame \\ Notre Dame, IN 46556, USA \\ Department of Mathematics, Purdue University \\ West Lafayette, IN 47907, USA \\ Email: mjh@math.mit.edu, palmieri@member.ams.org, jhs@math.purdue.edu
}

\section{Abstract}

We show that in a generalized Adams spectral sequence, the presence of a vanishing line of fixed slope (at some term of the spectral sequence, with some intercept) is a generic property.

\section{AMS Classification numbers Primary: 55T15}

Secondary: $55 \mathrm{P} 42$

Keywords: Adams spectral sequence, vanishing line, generic

Proposed: Haynes Miller

Seconded: Ralph Cohen, Gunnar Carlsson
Received: 17 March 1998

Revised: 27 May 1999

\section{Copyright Geometry and Topology}




\section{Introduction}

Our main result, Theorem 1.3, was motivated by several questions.

The thick subcategory theorem of Hopkins and Smith [4, Theorem 7] describes some important structure in stable homotopy theory: one can get a good deal of information about a finite $p$-local spectrum $X$ by knowing its "type" - that is, knowing the unique number $n$ so that its $n$th Morava $K$-theory $K(n)_{*} X$ is nonzero while $K(n-1)_{*} X=0$. While one cannot in general determine the type of a finite $p$-local spectrum by knowing just its mod $p$ cohomology, one might ask whether the type is reflected somehow in the behavior of the classical Adams spectral sequence. Our main theorem shows that the answer is yes, and we explain this behavior (the existence of a vanishing line with a particular slope) as our first application after the statement of the theorem.

Spectral sequences are often sequences of bigraded abelian groups $\left\{E_{r}^{* *}\right\}$, indexed starting at $r=1$ or $r=2$. The $E_{r+1}$-term is a subquotient of the $E_{r}$-term, so if one has some sort of vanishing result at $E_{r}$, such as " $E_{r}^{s, t}=0$ when $s \geq 3 t+4$ ", then the same result holds at $E_{r+k}$ for all $k \geq 0$. As one might imagine, such results can be quite useful. In a typical spectral sequence, one does not have an explicit description of $E_{r}$ when $r \geq 3$, so most vanishing theorems have been proven at the $E_{1}-$ or $E_{2}$-term; the main result of [5] is a good example of this. One might ask how to produce vanishing lines at later terms of a spectral sequence. Our main theorem provides a way of doing this; see the second and third applications after the statement of the theorem for examples.

The following definition is at the heart of the paper.

Definition 1.1 A property $P$ of spectra is generic if

- whenever a spectrum $X$ satisfies $P$, then so does any retract of $X$; and

- if $X \rightarrow Y \rightarrow Z$ is a cofibration and two of $X, Y$ and $Z$ satisfy $P$, then so does the third.

In other words, a property is generic if the full subcategory of all spectra satisfying it is thick.

For example, for any spectrum $E$, the property that $E_{*}(X)=0$ is a generic property of $X$.

We recall three other concepts: first, given a connective spectrum $W$, we write $|W|$ for its connectivity; that is, $|W|$ is the largest number $w$ so that $\pi_{n} W=0$ 
when $n \leq w$. Second, we assume that our ring spectrum $E$ satisfies the standard assumptions for the construction and convergence of the $E$-based Adams spectral sequence -in other words, the assumptions necessary for Theorem 15.1(iii) in [1, Part III]; see also Assumptions 2.2.5(a)-(c) and (e) in [8]. More precisely, we assume that $E$ satisfies the following.

\section{Condition 1.2}

- $E$ is a ring spectrum, associative and commutative up to homotopy.

- $\pi_{r} E=0$ for $r<0$.

- The map $\pi_{0} E \otimes \pi_{0} E \rightarrow \pi_{0} E$, induced by multiplication on $E$, is an isomorphism.

- Let $R$ be the largest subring of the rationals to which the ring map $\mathbf{Z} \rightarrow \pi_{0} E$ extends; then $H_{n}(E ; R)$ is finitely generated over $R$ for all $n$.

Third, in order to state the main theorem precisely, we need to work with $E$-complete spectra: " $E$-complete" is a technical condition on a spectrum $X$ which ensures that the $E$-based Adams spectral sequence converges to $\pi_{*} X$. We give a careful definition below (Definition 3.1); for now, we point out that if the spectrum $X$ is connective, then $E$-completeness is a mild restriction. For example, if $\pi_{0} E=\mathbf{Z}$, then every connective $X$ is $E$-complete; if $\pi_{0} E=\mathbf{Z}_{(p)}$, then every connective $p$-local $X$ is $E$-complete. See [1], [8], and [2] for more information.

This is our main result.

Theorem 1.3 Let $E$ be a ring spectrum satisfying Condition 1.2, and consider the $E$-based Adams spectral sequence $E_{*}^{* *}(X) \Rightarrow \pi_{*}(X)$. Fix a number $m$. The following properties of an $E$-complete spectrum $X$ are generic:

(i) There exist numbers $r$ and $b$ so that for all $s$ and $t$ with $s \geq m(t-s)+b$, then $E_{r}^{s, t}(X)=0$.

(ii) There exist numbers $r$ and $b$ so that for all finite spectra $W$ and for all $s$ and $t$ with $s \geq m(t-s-|W|)+b$, then $E_{r}^{s, t}(X \wedge W)=0$.

\section{Remark 1.4}

(a) One usually draws Adams spectral sequences $E_{r}^{s, t}$ with $s$ on the vertical axis and $t-s$ on the horizontal; in terms of these coordinates, the properties say that $E_{r}^{s, t}$ is zero above a line of slope $m$, with $s$-intercept $b$ in (i), and $s$-intercept $b-m|W|$ in (ii). 
(b) Assuming that $X$ is $E$-complete ensures that the spectral sequence converges, which we need to prove the theorem. We do not need to identify the $E_{2}$-term of the spectral sequence, so we do not need to know that $E$ is a flat ring spectrum - one of the other standard assumptions on ring spectra in discussions of the Adams spectral sequence.

We mention three applications of the theorem. Since for each prime $p$ there is a classification of the thick subcategories of the category of finite $p$-local spectra (see [4, Theorem 7$]$ ), then in this setting one may be able to identify all spectra with vanishing line of a given slope. Consider, for example, the classical mod $p$ Adams spectral sequence. This is based on the ring spectrum $E=H \mathbf{F}_{p}$, and every finite $p$-local torsion spectrum is $H \mathbf{F}_{p}$-complete. When $p=2$, for instance, since the mod 2 Moore spectrum has a vanishing line of slope $\frac{1}{2}$ at the $E_{2}$-term, then the mod $2^{n}$ Moore spectrum, and indeed any type 1 spectrum, has a vanishing line of slope $\frac{1}{2}$ at some $E_{r}$-term. Similarly, part of the proof of the thick subcategory theorem is the construction of a type $n$ spectrum with a vanishing line of slope $\frac{1}{2^{n+1}-2}$ at the $E_{2}$-term, and hence any type $n$ spectrum has a vanishing line of slope $\frac{1}{2^{n+1}-2}$ at some $E_{r}$-term. At odd primes, any type $n$ spectrum has a vanishing line of slope $\frac{1}{2 p^{n}-2}$ at some $E_{r}$-term of the classical mod $p$ Adams spectral sequence. Theorem 1.3 gives no control over the term $r$ or the intercept $b$ of the vanishing line.

Since the proof of Theorem 1.3 is formal, it also applies in any category which satisfies the axioms of a stable homotopy category, as given in [3]. The second author has used this result in an appropriate category of modules over the Steenrod algebra to prove a version of Quillen stratification for the cohomology $\operatorname{Ext}_{A}^{* *}\left(\mathbf{F}_{2}, \mathbf{F}_{2}\right)$ of the mod 2 Steenrod algebra $A$ (see [6]). A key part of the argument is to show that for a particular ring $R$, there is a map $\phi: \operatorname{Ext}_{A}^{* *}\left(\mathbf{F}_{2}, \mathbf{F}_{2}\right) \rightarrow R$ satisfying these two properties:

- Every element in $\operatorname{ker} \phi$ is nilpotent.

- For every element $y$ in $R$, there is an $n$ so that $y^{2^{n}} \in \operatorname{im} \phi$.

To prove this, one uses a certain generalized Adams spectral sequence in the setting of $A$-modules. This spectral sequence converges to $\operatorname{Ext}_{A}^{* *}\left(\mathbf{F}_{2}, \mathbf{F}_{2}\right)$, and the ring $R$ is the zero line of the $E_{2}$-term. The map $\phi$ is then the edge homomorphism. To prove that $\phi$ has the desired properties, one uses Theorem 1.3 together with some specific computations to show that for any $m>0$, the spectral sequence eventually has a vanishing line of slope $m$. Every element in $\operatorname{ker} \phi$ is represented by an element above the zero line, and hence must be nilpotent - its powers lie along a line of some slope, and some $E_{r}$-term has a 
vanishing line of smaller slope. For every $y \in R$, one can show that for all $n$, the targets of the possible differentials on $y^{2^{n}}$ all lie above a certain line of positive slope, and since some $E_{r}$-term has a vanishing line of smaller slope, then $y^{2^{n}}$ is a permanent cycle for all sufficiently large $n$. See $[6,7]$ for details.

A third application is the computation of localized homotopy groups. Suppose that $E$ is a ring spectrum satisfying Condition 1.2, and fix $v \in \pi_{*}(E)$. Say that an $E$-complete spectrum $X$ has a "nice $v$-action" if $X$ has a self-map which induces multiplication by some power of $v$ on $E_{*} X$, and if there is an $n$ so that the $E$-based Adams spectral sequence converging to $\pi_{*} X$ is a spectral sequence of $\pi_{0}(E)\left[v^{n}\right]$-modules, compatibly with the $v$-self map on $X$. Note that multiplication by $v^{n}$ does not increase Adams filtration: it acts "horizontally" in this spectral sequence. Given a spectrum $X$ with a nice $v$ action, if one inverts $v^{n}$ in the $E$-based Adams spectral sequence $E_{*}^{* *}(X)$, one gets a localized spectral sequence $v^{-1} E_{*}^{* *}(X)$. A natural question is, can one compute $v^{-1} \pi_{*} X$ from the localized spectral sequence? It is not hard to show that if the unlocalized spectral sequence $E_{*}^{* *}(X)$ has a horizontal vanishing line at some $E_{r}$-term, then the localized spectral sequence will converge to $v^{-1} \pi_{*} X$ : the vanishing line forces both the differentials and the extensions to behave well. Hence if one can find a spectrum $X$ for which $E_{r}^{* *}(X)$ has a horizontal vanishing line, one can conclude from Theorem 1.3 that for all spectra with nice $v$-actions in the thick subcategory generated by $X$, the localized spectral sequence converges and computes the localized homotopy groups.

\section{Composites of maps in towers}

In this section, we prove a lemma about genericity and composites of maps in towers. In the next section, we will apply this lemma to the $E$-based Adams tower to prove Theorem 1.3.

Let $F$. be an exact functor from spectra to towers of spectra; in other words, given a spectrum $X$, we have a diagram, functorial in $X$ :

$$
\cdots \rightarrow F_{n+1} X \rightarrow F_{n} X \rightarrow F_{n-1} X \rightarrow \cdots \text {. }
$$

By "exact", we mean that if $X \rightarrow Y \rightarrow Z$ is a cofibration, then so is $F_{n} X \rightarrow$ $F_{n} Y \rightarrow F_{n} Z$ for each $n$.

We want to show that the following property is generic in $X$ : all $r$-fold composites of maps in the tower $F_{\bullet} X$ are zero on homotopy, ie, are null after composition with any map from a sphere. Actually, we refine this condition in 
two ways: first, rather than using spheres as test spectra, we use an arbitrary set of spectra; and second, we only look at $r$-fold composites $F_{s+r} X \rightarrow F_{s} X$ when $s$ satisfies an inequality which may depend on the particular test spectra used.

Lemma 2.1 Fix a number $m$ and an exact functor $F_{\bullet}$ from spectra to towers of spectra. Let $\mathcal{T}=\left\{\left(W_{\alpha}, n_{\alpha}\right)\right\}$ be a set of pairs of the form (spectrum, number). The following condition is generic in $X$ :

(*) There exist numbers $r$ and $b$ so that for all $\left(W_{\alpha}, n_{\alpha}\right) \in \mathcal{T}$, all $s$ with $s \geq m n_{\alpha}+b$, and all degree zero maps $W_{\alpha} \rightarrow F_{s+r} X$, the composite $W_{\alpha} \rightarrow F_{s+r} X \rightarrow F_{s} X$ is null.

In the next section, we will apply this in the two cases

$$
\begin{gathered}
\mathcal{T}=\left\{\left(S^{n}, n\right): n \in \mathbf{Z}\right\}, \\
\mathcal{T}=\{(W,-|D W|): W \text { finite }\}
\end{gathered}
$$

where $D W$ is the Spanier-Whitehead dual of $W$.

Proof If $Y$ is a retract of $X$, then the tower $F_{\bullet} Y$ is a retract of $F_{\bullet} X$, so if $F_{s+r} X \rightarrow F_{s} X$ is null after mapping in each $W_{\alpha}$, then so is $F_{s+r} Y \rightarrow F_{s} Y$. (Given $W_{\alpha} \rightarrow F_{s+r} Y$, then consider

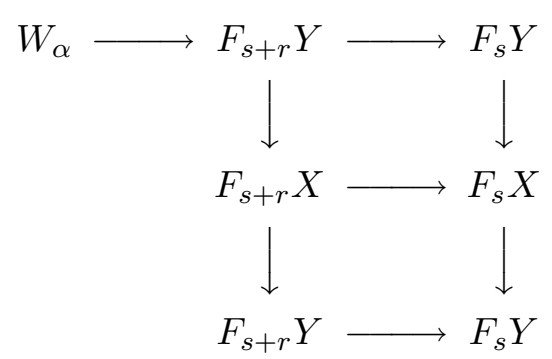

Then the composite $W_{\alpha} \rightarrow F_{s} Y$ factors through $W_{\alpha} \rightarrow F_{s+r} X \rightarrow F_{s} X$.)

The condition $(*)$ involves numbers $r$ and $b$, and we write $(*)_{r, b}$ if we want to specify the numbers.

Given a cofibration sequence $X \stackrel{f}{\rightarrow} Y \stackrel{g}{\rightarrow} Z$ in which $X$ and $Z$ satisfy conditions $(*)_{r, b}$ and $(*)_{r^{\prime}, b^{\prime}}$, respectively, we show that $Y$ satisfies $(*)_{r+r^{\prime}, \max \left(b, b^{\prime}-r\right)}$. Con- 
sider the following commutative diagram, in which the rows are cofibrations:

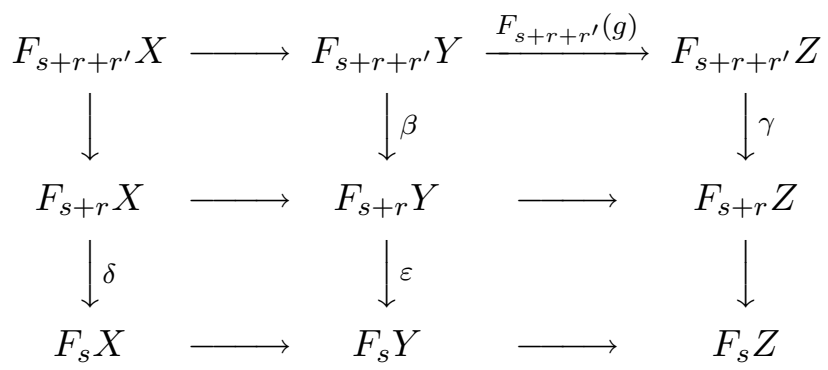

We fix $\left(W_{\alpha}, n_{\alpha}\right) \in \mathcal{T}$ and assume that $s \geq m n_{\alpha}+\max \left(b, b^{\prime}-r\right)$, so that we have

$$
\begin{gathered}
s \geq m n_{\alpha}+b, \\
s+r \geq m n_{\alpha}+b^{\prime} .
\end{gathered}
$$

Given any map $\zeta: W_{\alpha} \rightarrow F_{s+r+r^{\prime}} Y$, then since $\gamma \circ F_{s+r+r^{\prime}}(g) \circ \zeta$ is null, the composite $\beta \circ \zeta$ factors through $F_{s+r} X$, giving

$$
\zeta^{\prime}: W_{\alpha} \rightarrow F_{s+r} X
$$

Since $\delta \circ \zeta^{\prime}$ is null, though, then the composite

$$
W_{\alpha} \stackrel{\zeta}{\rightarrow} F_{s+r+r^{\prime}} Y \stackrel{\beta}{\rightarrow} F_{s+r} Y \stackrel{\varepsilon}{\rightarrow} F_{s} Y
$$

is null.

\section{Adams towers and the proof of Theorem 1.3}

The difficulty in proving a result like Theorem 1.3 is that the $E_{r}$-term of an Adams spectral sequence does not have nice exactness properties if $r \geq 3$ a cofibration of spectra does not lead to a long exact sequence of $E_{r}$-terms, for instance. So we prove the theorem by showing that the purported generic conditions are equivalent to other conditions on composites of maps in the Adams tower, and we apply Lemma 2.1 to conclude that those other conditions are generic.

We start by describing the standard construction of the Adams spectral sequence, as found in [1, III.15], [8, 2.2], and any number of other places. Given a ring spectrum $E$, we let $\bar{E}$ denote the fiber of the unit map $S^{0} \rightarrow E$. For any integer $s \geq 0$, we let

$$
\begin{gathered}
F_{s} X=\bar{E}^{\wedge s} \wedge X, \\
K_{s} X=E \wedge \bar{E}^{\wedge s} \wedge X .
\end{gathered}
$$


We use these to construct the following diagram of cofibrations, which we call the Adams tower for $X$ :

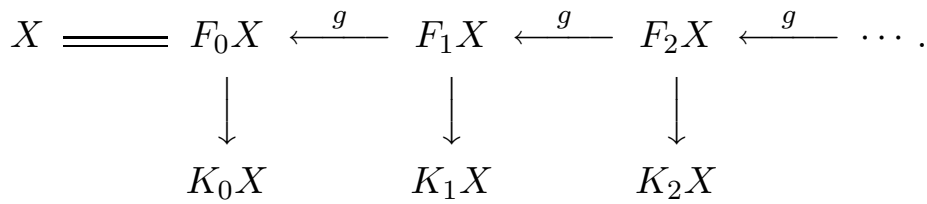

This construction satisfies the definition of an " $E_{*}-$ Adams resolution" for $X$, as given in $[8,2.2 .1]$ - see $[8,2.2 .9]$. Note also that $F_{s} X=X \wedge F_{s} S^{0}$, and the same holds for $K_{s} X$ - the Adams tower is functorial and exact.

We pause to define $E$-completeness.

Definition 3.1 A spectrum $X$ is $E$-complete if the inverse limit of its Adams tower is contractible.

Given the Adams tower for $X$, if we apply $\pi_{*}$, we get an exact couple and hence a spectral sequence. This is called the E-based Adams spectral sequence. More precisely, we let

$$
\begin{aligned}
& D_{1}^{s, t}=\pi_{t-s} F_{s} X, \\
& E_{1}^{s, t}=\pi_{t-s} K_{s} X .
\end{aligned}
$$

If we let $g: F_{s+1} X \rightarrow F_{s} X$ denote the natural map, then $g_{*}=\pi_{t-s}(g)$ is the map $D_{1}^{s+1, t+1} \rightarrow D_{1}^{s, t}$. Then we have the following exact couple (the pairs of numbers indicate the bidegrees of the maps):

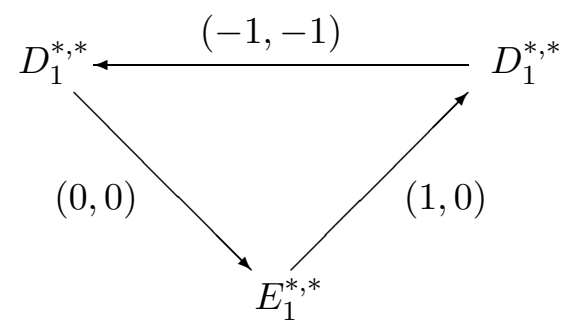

This leads to the following $r$ th derived exact couple, where $D_{r}^{s, t}$ is the image of $g_{*}^{r-1}$, and the map $D_{r}^{s+1, t+1} \rightarrow D_{r}^{s, t}$ is the restriction of $g_{*}$ :

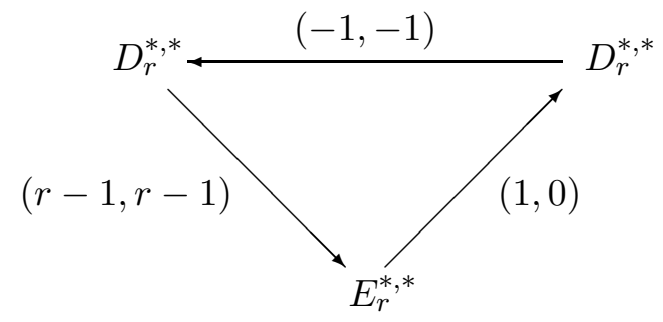

Geometry and Topology, Volume 3 (1999) 
Unfolding the $r$ th derived exact couple leads to the following exact sequence:

$$
\cdots \rightarrow E_{r}^{s, t+1} \rightarrow D_{r}^{s+1, t+1} \rightarrow D_{r}^{s, t} \rightarrow E_{r}^{s+r-1, t+r-1} \rightarrow \cdots
$$

Fix a number $m$. With respect to the $E$-based Adams spectral sequence $E_{*}^{* *}(-)$, we have the following conditions on a spectrum $X$ :

(1) There exist numbers $r$ and $b$ so that for all $s$ and $t$ with $s \geq m(t-s)+b$, then $D_{r}^{s, t}(X)=0$. (In other words, the map $g_{*}^{r-1}: \pi_{t-s}\left(F_{s+r-1} X\right) \rightarrow$ $\pi_{t-s}\left(F_{s} X\right)$ is zero. In other words, for all maps $f: S^{t-s} \rightarrow F_{s+r-1} X$, the composite $g^{r-1} \circ f$ is null.)

(2) There exist numbers $r$ and $b$ so that for all $s$ and $t$ with $s \geq m(t-s)+b$, then $E_{r}^{s, t}(X)=0$.

(3) There exist numbers $r$ and $b$ so that for all finite spectra $W$, all $s$ with $s \geq-m|D W|+b$, and all maps $W \rightarrow F_{s+r-1} X$, then the composite $W \rightarrow$ $F_{s+r-1} X \rightarrow F_{s} X$ is null. (Here, $D W$ denotes the Spanier-Whitehead dual of $W$. .)

(4) There exist numbers $r$ and $b$ so that for all finite spectra $W$ and for all $s$ and $t$ with $s \geq m(t-s-|W|)+b$, then $E_{r}^{s, t}(X \wedge W)=0$.

As with the condition in the proof of Lemma 2.1, each condition involves a pair of numbers $r$ and $b$, and we write $(1)_{r, b}$ to indicate condition (1) with the numbers specified, and so forth.

If $m=0$, for instance, then condition (3) says that $F_{s+r-1} X \rightarrow F_{s} X$ is a phantom map whenever $s \geq b$. If $m=0$, then condition (1) says that $F_{s+r-1} X \rightarrow F_{s} X$ is a ghost map (zero on homotopy) whenever $s \geq b$.

Lemma 3.3 Fix a spectrum $X$, and fix numbers $m, r$, and $b$. We have the following implications:

(a) If $r \geq-m$, then $(1)_{r, b} \Rightarrow(2)_{r, b+r-1}$. If $r<-m$, then $(1)_{r, b} \Rightarrow$ $(2)_{r, b+m-1}$.

(b) Suppose that $X$ is $E$-complete. If $r \geq 1-m$, then $(2)_{r, b} \Rightarrow(1)_{r, b+m}$. If $r<1-m$, then $(2)_{r, b} \Rightarrow(1)_{r, b-r+1}$.

(c) If $r \geq-m$, then $(3)_{r, b} \Rightarrow(4)_{r, b+r-1}$. If $r<-m$, then $(3)_{r, b} \Rightarrow$ $(4)_{r, b+m-1}$.

(d) Suppose that $X$ is $E$-complete. If $r \geq 1-m$, then $(4)_{r, b} \Rightarrow(3)_{r, b+m}$. If $r<1-m$, then $(4)_{r, b} \Rightarrow(3)_{r, b-r+1}$.

(Obviously, $(3)_{r, b} \Rightarrow(1)_{r, b}$ and $(4)_{r, b} \Rightarrow(2)_{r, b}$, but we do not need these facts.) 
Proof As above, we write $g$ for the map $F_{s+1} X \rightarrow F_{s} X$ and $g_{*}$ for the map $D_{1}^{s+1, t+1} \rightarrow D_{1}^{s, t}$, so that $D_{r}^{s, t}$ is the image of

$$
g_{*}^{r-1}: \pi_{t-s} F_{s+r-1} X \rightarrow \pi_{t-s} F_{s} X .
$$

(a) Assume that if $s \geq m(t-s)+b$, then $D_{r}^{s, t}=0$. In the case $r \geq-m$, if $s \geq m(t-s)+b$, then $s+r \geq m((t+r-1)-(s+r))+b$; so we see that $D_{r}^{s+r, t+r-1}=0$. By the long exact sequence (3.2), we conclude that $E_{r}^{s+r-1, t+r-1}=0$ when $s \geq m(t-s)+b$. Reindexing, we find that $E_{r}^{p, q}=0$ when $p \geq m(q-p)+b+r-1$; ie, condition $(2)_{r, b+r-1}$ holds. The case $r<-m$ is similar; in this case, the long exact sequence implies that $E_{r}^{s-1, t}=0$.

(b) Assume that $r \geq 1-m$. If $E_{r}^{s, t}(X)=0$ whenever $s \geq m(t-s)+b$, then $E_{r}^{s+r-1, t+r-2}(X)=0$ when $s \geq m(t-s)+b$. So by the exact sequence (3.2), we see that $D_{r}^{s+1, t} \rightarrow D_{r}^{s, t-1}$ is an isomorphism under the same condition. This map is induced by $g_{*}: \pi_{t-s-1} F_{s+1} X \rightarrow \pi_{t-s-1} F_{s} X$, so we conclude that when $s \geq m(t-s)+b$, we have

$$
\begin{gathered}
\lim _{q} \pi_{t-s-1} F_{q} X=D_{r}^{s, t-1}, \\
\lim _{q}^{1} \pi_{t-s-1} F_{q} X=0 .
\end{gathered}
$$

But since $X$ is $E$-complete, then $\varliminf_{q} \pi_{t-s-1} F_{q} X=0$, so $D_{r}^{s, t-1}=\operatorname{im} g_{*}^{r-1}=$ 0 . Reindexing gives $D_{r}^{p, q}=0$ when $p \geq m(q+1-p)+b$; ie, $(2)_{r, b}$ implies $(1)_{r, b+m}$.

If $r<1-m$, then a similar argument shows that $D_{r}^{s-r+1, t-r+1}=0$.

Parts (c) and (d) are similar.

Proof of Theorem 1.3 This follows immediately from Lemmas 3.3 and 2.1. More precisely, to show that condition (i) is generic, one applies Lemma 2.1 to the set $\mathcal{T}=\left\{\left(S^{n}, n\right): n \in \mathbf{Z}\right\}$. For condition (ii), one applies it to the set $\mathcal{T}=\{(W,-|D W|): W$ finite $\}$. 


\section{References}

[1] J F Adams, Stable homotopy and generalised homology, Chicago Lectures in Mathematics, University of Chicago Press, Chicago-London (1974)

[2] A K Bousfield, The localization of spectra with respect to homology, Topology, 18 (1979) 257-281

[3] M Hovey, J H Palmieri, and N P Strickland, Axiomatic stable homotopy theory, Mem. Amer. Math. Soc. 128 (1997), no. 610

[4] M J Hopkins, J H Smith, Nilpotence and stable homotopy theory II, Annals of Math. 148 (1998) 1-49

[5] H R Miller, C Wilkerson, Vanishing lines for modules over the Steenrod algebra, J. Pure Appl. Algebra, 22 (1981) 293-307

[6] J H Palmieri, Quillen stratification for the Steenrod algebra, Annals of Math. (1999) to appear

[7] J H Palmieri, Stable homotopy over the Steenrod algebra, Mem. Amer. Math. Soc. to appear

[8] D C Ravenel, Complex cobordism and stable homotopy groups of spheres, Academic Press, Orlando, Florida (1986) 